\title{
Tumor sólido pseudopapilar del páncreas: estudio descriptivo de una serie de casos*
}

\author{
DrS. MILENKO SLAKO M. ${ }^{1}$, CÉSAR MUÑOZ C. ${ }^{1}$, EDUARDO BRICEÑO V. ${ }^{1}$, FABRIZIO MOISAN P. ${ }^{1}$, \\ JORGE MARTÍNEZ C. ${ }^{1}$, JUAN CARLOS PATILLO S. ${ }^{1}$, NICOLÁS JARUFE C. ${ }^{1}$
}

1 Departamento de Cirugía Digestiva. Facultad de Medicina. Pontificia Universidad Católica de Chile. Santiago, Chile.

\begin{abstract}
Solid pseudopapilary tumors of the pancreas: descriptive study of a case series

Background: Rare neoplasm that occurs most often in young women, neoplasm with low degree of malignancy. Aim: to describe the clinical characteristics and postoperative results and long-term in a series of patients. Material and Methods: Descriptive study of patients with confirmed histological diagnosis operated at our hospital between January 2000 and June 2011. Biodemographical, preoperative, clinical, surgical technique and postoperative results were recorded. Results: 11 patients, ten of them female, with an average age of 28 years (14-43 years). The most common symptom was abdominal pain. Nine patients had lesions of the body and tail of pancreas and 2 patients had lesions in head of pancreas. Corporo-caudal pancreatectomia were done in 9 cases (6 laparoscopic surgeries) and 2 pancreatoduodenectomy (1 totally laparoscopic). The average size of tumors was $5.5 \mathrm{~cm}(2.2-13 \mathrm{~cm})$, the margin was negative in all cases and no positive lymph nodes. Six patients had a complication: 4 pancreatic fistulas (type A) which were controlled with drainage installed during surgery. One patient had postoperative abdominal collection requiring laparoscopic drainage. There was no operative mortality in this series. The average follow-up was 21 months (5-93 months). There was no recurrence or mortality at the end of follow-up. Conclusions: Is an uncommon neoplasm and occurs primarily in young women. The complete surgical resection is the best treatment, and can be done by laparoscopy surgery with low morbidity.
\end{abstract} (MeSH).

Key words: Pancreatic neoplasms (MeSH), solid pseudopapillary pancreatic tumor, laparoscopy

\section{Resumen}

Introducción: Es una neoplasia poco frecuente, con bajo grado de malignidad, se presenta principalmente en mujeres jóvenes. El objetivo de este estudio es describir las características clínicas y los resultados postoperatorios a corto y largo plazo de una serie de pacientes intervenidos en nuestro centro. Material y Método: Estudio descriptivo de pacientes con diagnóstico histológico confirmado, operados en nuestro hospital entre enero de 2000 y junio de 2011. Se consignaron variables biodemográficas, estudio preoperatorio, manifestaciones clínicas, técnica quirúrgica y resultados postoperatorios. Resultados: La serie está constituida

*Recibido el 16 de agosto de 2011 y aceptado para publicación el 12 de diciembre de 2011.

Correspondencia: Dr. Nicolás Jarufe C.

Marcoleta \#350. Santiago. Chile.

njarufe@med.puc.c 
por 11 pacientes, diez de ellas de sexo femenino y con una edad promedio de 28 años (14-43 años). El síntoma más frecuente fue el dolor abdominal. Nueve pacientes presentaron lesiones córporocaudales del páncreas y 2 tumores de la cabeza del páncreas. Se realizaron 9 pancreatectomías córporocaudales (6 laparoscópicas) y 2 duodenopancreatectomías (1 totalmente laparoscópica). El tamaño promedio de los tumores fue 5,5 cm (2,2-13 cm), el margen fue negativo en todos ellos y no había linfonodos positivos. Seis pacientes presentaron alguna complicación: 4 fístulas pancreáticas (tipo A) que fueron controladas con el drenaje instalado durante la cirugía. Un paciente presentó una colección abdominal postoperatoria que requirió de drenaje laparoscópico. No hubo mortalidad operatoria en esta serie. El seguimiento promedio fue de 21 meses (5-93 meses). No hubo recurrencia ni mortalidad al cierre del seguimiento. Conclusión: El TSP es una neoplasia infrecuente. La resección quirúrgica completa es el tratamiento de elección, puede ser laparoscópica.

Palabras clave: Neoplasias pancreáticas, tumor sólido seudopapilar pancreático, laparoscopia.

\section{Introducción}

El tumor sólido pseudopapilar del páncreas (TSP) es también conocido como tumor sólido quístico del páncreas, tumor de Frantz (en honor al primer reporte) $)^{1}$, tumor de Hamoudi (caracterización del tumor)², tumor quístico papilar, tumor sólido papilar o neoplasia epitelial papilar ${ }^{3}$. Este tumor es una neoplasia poco frecuente y constituye aproximadamente el 1-2\% de todas las neoplasias del páncreas ${ }^{4}$. Se presenta principalmente en mujeres durante la segunda o tercera década de la vida ${ }^{5,6}$.

Es considerada una neoplasia de bajo potencial de malignidad, dado por la posibilidad de invasión local por contigüidad, y bajo poder de diseminación metastásica ${ }^{7,8}$. El tratamiento quirúrgico es la única alternativa curativa para este tipo de tumores, sin embargo, la resección incompleta se asocia a recurrencia y disminución de la sobrevida ${ }^{9}$.

El objetivo de este estudio es describir las características clínicas y los resultados postoperatorios de una serie de pacientes con TSP intervenidos quirúrgicamente en un Hospital Clínico Universitario.

\section{Material y Método}

Diseño del estudio: Estudio descriptivo tipo serie de casos.

Lugar y período del estudio: Este estudio se realizó en el Hospital Clínico de la Pontificia Universidad Católica de Chile entre enero de 2000 y junio de 2011, período en el cual se ha constituido una base de datos con los pacientes intervenidos quirúrgicamente por patología pancreática.

Criterios de inclusión: Pacientes intervenidos quirúrgicamente por una lesión tumoral pancreática y cuyo estudio histopatológico fue compatible con un TSP.

Criterios de exclusión: No contemplados en este estudio.

Muestreo: No probabilístico de casos consecutivos y por conveniencia.
Variables estudiadas: Biodemográficas, características clínicas, estudio imagenológico preoperatorio (Ecografía abdominal (US), tomografía computada abdominal con contraste (TC) y resonancia nuclear magnética abdominal con contraste (RNM)), técnica y vía de abordaje de la resección pancreática. La morbilidad postoperatoria se clasificó según lo propuesto por Dindo D et $\mathrm{al}^{10}$, y en particular la fístula pancreática se clasificó de acuerdo a la clasificación de la International Study Group of Pancreatic Fitulae (ISGPF) ${ }^{11}$.

Estudio histopatológico: El análisis de la pieza de resección fue realizado por un médico anatomopatológo con experiencia en el estudio de lesiones neoplásicas del aparato digestivo.

Seguimiento postoperatorio: El seguimiento de los pacientes se realizó en base a controles ambulatorios programados y exámenes de laboratorio e imagenológicos a demanda clínica del médico tratante.

Análisis estadístico: El análisis estadístico de este estudio se realizó en base a estadística descriptiva con medidas de tendencia central y dispersión. Para este análisis se utilizó el paquete estadístico STATA 10.0 .

Reporte: La preparación y redacción de este manuscrito se ha realizado según las recomendaciones de la iniciativa STROBE para el reporte de estudios observacionales $^{12}$.

\section{Resultados}

En el período de estudio se realizaron 171 cirugías resectivas pancreáticas. En 11 casos $(6,4 \%)$ el estudio histopatológico fue compatible con un TSP. La serie se constituyó por 10 mujeres y un hombre, la mediana de edad fue 28 años (14-43 años).

La presentación clínica más frecuente fue el dolor abdominal en 8 casos. Un paciente presentó una pancreatitis aguda que motivó su estudio imagenológico; un paciente fue diagnosticado incidentalmente en el estudio por una patología de origen 
ginecológico y en una paciente fue un hallazgo en una cirugía de gastrectomía en manga laparoscópica. Ningún paciente presentó ictericia al momento del diagnóstico.

El estudio preoperatorio se realizó con TC o RNM que evidenció las lesiones sólidas, quísticas o mixtas del páncreas en la totalidad de la serie (Figuras 1 y 2). En 9 pacientes (80\%) esta lesión se encontró en el cuerpo o la cola del páncreas. El estudio imagenológico preoperatorio fue concordante con la histología en 6 casos. Los diagnósticos radiológicos se resumen en la Tabla 1.

Respecto al tratamiento quirúrgico, la resección tumoral de los TSP ubicados en la cabeza del páncreas (2 pacientes) se realizó a través de una pancreatoduodenectomía cefálica (PDC), una de ellas totalmente laparoscópica. Para el tratamiento de los restantes 8 casos con tumores en cuerpo y cola se realizaron 4 pancreatectomías corporocaudales por vía abierta, una de las cuales fue por conversión de una cirugía laparoscópica (Figura 3) y 5 pancreatectomías corporocaudales laparoscópicas (3 con técnica de Warshaw).

Seis pacientes presentaron algún tipo de morbilidad postoperatoria: 4 fístulas pancreáticas tipo A (Tipo I), un infarto esplénico (Tipo II); y una colección intraabdominal tardía que se resolvió por laparoscopia (Tipo IIIB). El resumen de las complicaciones se resume en la Tabla 2.

El tamaño tumoral promedio fue de $5,5 \mathrm{~cm}$ (rango: 2,2-13 cm). Los bordes quirúrgicos fueron negativos en todos los pacientes y 1 paciente presentó infiltración del tejido retropancreático pero sin constituir un borde microscópico positivo. Se resecó un promedio de 5 linfonodos (rango: 1-16

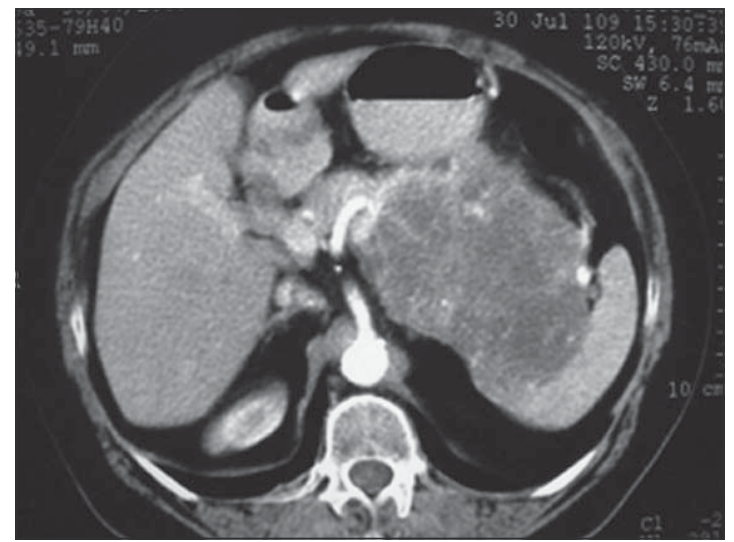

Figura 1. Tomografía abdominal con contraste endovenoso en fase precoz que muestra lesión quística compleja que compromete cuerpo y cola de páncreas.

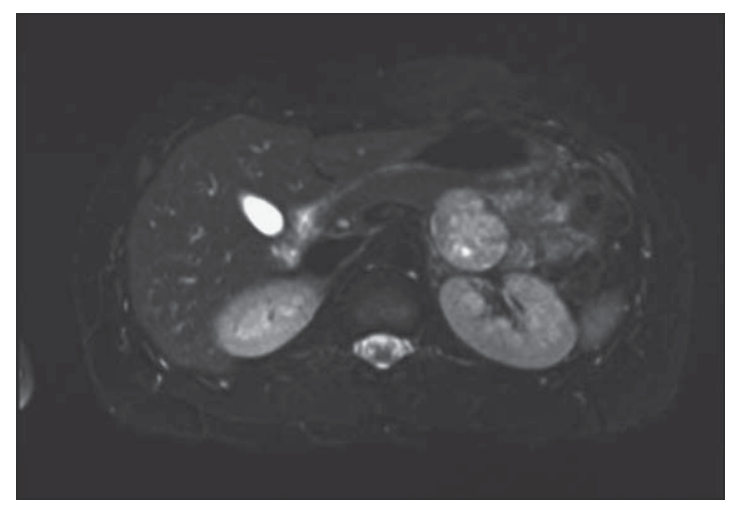

Figura 2. Resonancia nuclear magnética en T2 que muestra lesión microquística del cuerpo pancreático.

Tabla 1. Características y diagnósticos imagenológicos preoperatorios

\begin{tabular}{|clll|}
\hline Paciente & Ubicación & Características al TC o RNM & Diagnóstico preoperatorio \\
\hline 1 & Cabeza & Lesión quística compleja & Cistoadenoma mucinoso \\
\hline 2 & Cabeza & Lesión quística compleja & Cistoadenoma seroso \\
\hline 3 & Cuerpo & Lesión microquística & Cistoadenoma seroso \\
\hline 4 & Cuello-cuerpo & Masa sólido quística & Cistoadenocarcinoma mucinoso \\
\hline 5 & Cuerpo-cola & Masa sólido quística & TSP \\
\hline 6 & Cuello-cuerpo & Lesión quística compleja & TSP \\
\hline 8 & Cuerpo-cola & Lesión sólido quística & TSP \\
\hline 9 & Cuerpo & Tumor sólido exofítico & TSP \\
\hline 10 & Cola & Tumor quístico complejo & TSP \\
\hline 11 & Cola & Lesión sólida & Tumor neuroendocrino \\
\hline
\end{tabular}

TC: Tomografía computada, RNM: Resonancia magnética, TSP: Tumor pseudopapilar de páncreas. 


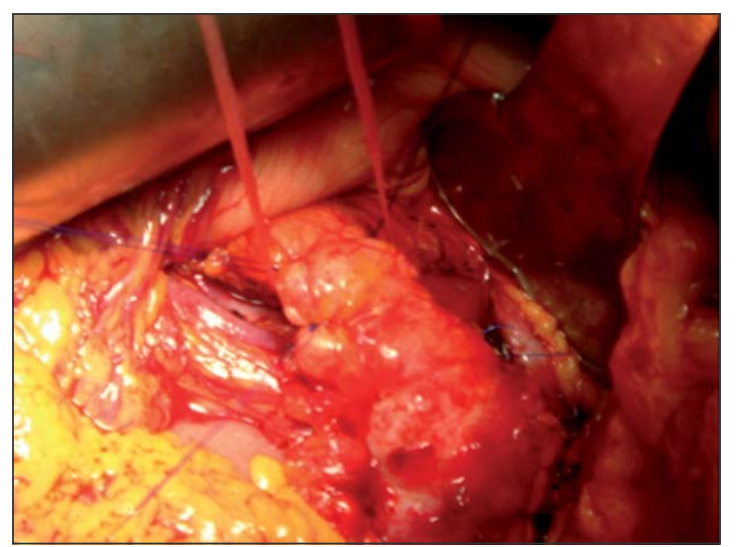

Figura 3. Visión macroscópica in situ de una lesión sólida pseudopapilar de la cola del páncreas.

\section{Tabla 2. Morbilidad operatoria y tratamiento realizado}

\begin{tabular}{|rrlcl|}
\hline P & EH & Morbilidad & $\begin{array}{c}\text { Clasificación } \\
\text { Clavien-Dindo }\end{array}$ & Tratamiento \\
\hline 1 & 14 & No & 0 & \\
2 & 11 & No & 0 & \\
3 & 7 & No & 0 & \\
4 & 9 & FP tipo A & I & Médico \\
\hline 5 & 11 & No & 0 & \\
6 & 5 & FP tipo A & I & Médico \\
\hline 7 & 6 & Colección & IIIB & Drenaje \\
& & abdominal & & laparoscópico \\
8 & 6 & Infarto & II & Médico \\
\hline 9 & 7 & No & 0 & \\
\hline 10 & 6 & FP tipo A & I & Médico \\
\hline 11 & 6 & FP tipo A & I & Médico \\
\hline
\end{tabular}

$\mathrm{P}=$ paciente. $\mathrm{EH}=$ Estadía hospitalaria (días). FP = Fístula pancreática.

linfonodos) y ninguno de ellos presentó compromiso tumoral metastásico.

Se realizó seguimiento a todos los pacientes con una mediana de 21 meses (1-93 meses). Al momento del cierre del estudio, no hay evidencias de recidiva locoregional o de metástasis a distancia en la totalidad de la serie. Un paciente desarrolló diabetes mellitus durante este período y actualmente se encuentra en tratamiento por esta patología, en tanto que, otro paciente presentó diarrea crónica secundaria a insuficiencia pancreática exócrina y se encuentra en tratamiento con enzimas pancreáticas con buena respuesta clínica.

\section{Discusión}

El TSP, nombre por el cual fue definido este tumor en el año 1996 por la Organización Mundial de la Salud, es una neoplasia que ha despertado un especial interés en los últimos años y sobre la cual ha visto un aumento en los reportes, al igual que otras lesiones quísticas del páncreas. Este fenómeno probablemente no corresponde a un aumento real de su incidencia ${ }^{5,6}$, sino más bien, parece estar relacionado a la mayor conciencia sobre la necesidad del diagnóstico precoz de la patología tumoral pancreática en general, así como la mayor disponibilidad y mejoría en los métodos de estudio por imágenes ${ }^{11,12}$.

En nuestro país, el conocimiento respecto a esta neoplasia se limita a reportes de casos y esta es la mayor serie con seguimiento comunicada en la población chilena ${ }^{13-17}$.

En la actualidad aún existen interrogantes respecto a la biología tumoral e histopatogénesis de esta neoplasia, sin embargo, sabemos que algunas mutaciones en genes específicos así como la expresión en moléculas de adhesión celular y señalización intercelular, podrían estar asociadas a factores pronósticos y de la evolución en esta neoplasia ${ }^{18-20}$. Otro aspecto sobre el cual ya existe algún grado de evidencia es el efecto de la influencia hormonal, la cual jugaría un rol en el desarrollo y crecimiento del TSP, esto se fundamenta en la mayor proporción de esta neoplasia en el sexo femenino (unas 8 veces más frecuente $)^{5,6,21}$; y en el resultado de estudios in vitro que muestran respuesta proliferativa a moléculas estrogénicas en líneas celulares ${ }^{22}$.

Su historia natural está aun pobremente definida. La manifestación clínica más frecuente es el dolor abdominal ${ }^{5,6}$, al igual que en nuestra serie, síntoma por el cual se inicia un estudio imagenológico. En nuestro estudio, un paciente presentó una pancreatitis aguda y en su estudio se evidenció la lesión pancreática. Esto ya ha sido reportado por otros autores $^{23,24}$, y confirma la necesidad de estudiar imagenológicamente los pacientes que presentan una pancreatitis aguda en ausencia de colecisto o coledocolitiasis. La localización del tumor en nuestros pacientes es de predominio en cuerpo y cola del páncreas, concordante con otras series reportadas s,6,21,25 $^{5}$

El diagnóstico puede ser hecho mediante TC o RNM con buena precisión, sin embargo, esta neoplasia puede presentar patrones que dificulten su diagnóstico imagenológico ${ }^{26,27}$. En algunos casos puede ser de utilidad la punción con aguja fina mediante endosonografía para el diagnóstico histológico preoperatorio, sin embargo, este procedimiento debiera ser de excepción, ya que con la calidad de estudios de imágenes actuales, el diagnóstico se puede realizar con procedimientos no invasivos ${ }^{28}$. 
Dado su potencial maligno y la ausencia de respuesta a otras terapias, su tratamiento consiste en la resección quirúrgica del tumor primario completa (R0), así como de los órganos comprometidos por invasión local o la enfermedad metastásica asocia$\mathrm{da}^{9,29}$.

El desarrollo de la cirugía laparoscópica ha permitido la resolución de la mayoría de nuestros pacientes por esta vía, con los beneficios que ella otorga principalmente tratándose de pacientes jóvenes, sin limitar el pronóstico oncológico. Este tipo de tumores, cuando se presentan en etapas iniciales y con menores tamaños son un candidato óptimo para esta vía de abordaje, dado el comportamiento de baja agresividad ${ }^{30,31}$. En caso de tumores de gran tamaño, la posibilidad de realizar una cirugía laparoscópica es más limitada y la vía abierta puede ser la primera alternativa desde el inicio ${ }^{32}$.

La fístula pancreática posterior a una pancreatectomía, ya sea cefálica o corporo-caudal sigue siendo un problema no resuelto dentro de la cirugía pancreática. Múltiples técnicas se han utilizado para tratar de disminuir esta complicación, sin embargo, todos estos han sido infructuosos. El uso rutinario de drenajes en el remanente pancreático permite controlar por esta vía las fístulas pancreáticas postoperatorias que se presentan; si bien esta práctica ha sido cuestionada ${ }^{33}$, la evidencia para no utilizar drenajes posterior a una resección pancreática aún es limitada ${ }^{34}$. En nuestra serie, el uso rutinario de drenajes postoperatorios nos permitió tratar en forma conservadora a los pacientes que presentaron fístulas pancreáticas, los cuales fueron dados de alta con drenaje y se controlaron en forma ambulatoria.

Dado el bajo porcentaje de linfonodos comprometidos y la ausencia de un valor pronóstico confirmado en ellos, no está indicada una linfadenectomía formal en estos pacientes, sin embargo, la resección debe ser R0 ya que el TSP presenta un pronóstico oncológico en general bueno; en esta serie todos los pacientes están libre de enfermedad y presentan sobrevida al momento del cierre del seguimiento. En pacientes con metástasis o recidiva hepática la sobrevida es prolongada (tanto con cirugía como sin ella), estando de todas maneras, recomendada la resección quirúrgica en estos casos.

Los tratamientos adyuvantes, ya sea quimioterapia, radioterapia u hormonoterapia, no han mostrado mayor beneficio en estos pacientes ${ }^{5,6,21}$.

Finalmente, esta patología debe ser sospechada en toda mujer joven que presente una masa sólida o sólido quística pancreática, con un estudio de imágenes compatible, siendo la cirugía laparoscópica una alternativa factible en casos seleccionados.

\section{Referencias}

1. Frantz VK. Tumors of the pancreas. Atlas of tumor pathology. Washington DC: Armed forces Institute of Pathology; 1959. p. 32-3.

2. Hamoudi AB, Misugi K, Grosfeld JL, Reiner CB. Papillary epithelial neoplasm of pancreas in a child. Report of a case with electron microscopy. Cancer 1970;26:1126-34.

3. Reddy S, Cameron JL, Scudiere J, Hruban RH, Fishman EK, Ahuja N, et al. Surgical management of solidpseudopapillary neoplasms of the pancreas (Franz or Hamoudi tumors): a large single-institutional series. J Am Coll Surg. 2009;208: 950-7; discussion 7-9.

4. Martin RC, Klimstra DS, Brennan MF, Conlon KC. Solid-pseudopapillary tumor of the pancreas: a surgical enigma? Ann Surg Oncol. 2002;9:35-40.

5. Yu PF, Hu ZH, Wang XB, Guo JM, Cheng XD, Zhang YL, et al. Solid pseudopapillary tumor of the pancreas: a review of 553 cases in Chinese literature. World J Gastroenterol. 2010;16:1209-14.

6. Papavramidis T, Papavramidis S. Solid pseudopapillary tumors of the pancreas: review of 718 patients reported in English literature. J Am Coll Surg. 2005;200: 965-72.

7. Kang CM, Kim KS, Choi JS, Kim H, Lee WJ, Kim BR. Solid pseudopapillary tumor of the pancreas suggesting malignant potential. Pancreas 2006;32:276-80.

8. Reano G, De Vinatea J, Kometter F, Villanueva L, Gonzales E, Uribe M, et al. Solid pseudopapillary tumor of the pancreas: a neoplasm of low malignant potential?. Rev Gastroenterol Peru 2011;31:61-71.

9. Campanile M, Nicolas A, LeBel S, Delarue A, Guys JM, de Lagausie P. Frantz's tumor: is mutilating surgery always justified in young patients? Surg Oncol. 2011;20:121-5.

10. Dindo D, Demartines N, Clavien PA. Classification of surgical complications: a new proposal with evaluation in a cohort of 6336 patients and results of a survey. Ann Surg. 2004;240:205-13.

11. Bassi C, Dervenis C, Butturini G, Fingerhut A, Yeo C, Izbicki J, et al. Postoperative pancreatic fistula: an international study group (ISGPF) definition. Surgery 2005;138:8-13.

12. Noah N. The STROBE initiative: STrengthening the Reporting of OBservational studies in Epidemiology (STROBE). Epidemiol Infect. 2008;136:865.

13. Acuña R, Soto P, Muñoz VJA. Tumor sólido pseudopapilar de páncreas, reporte de un caso. Rev Chil Pediatr. 2008;79:404-8.

14. De Aretxabala X, Rencoret G, Maluenda F, Fernández CA. Tumor sólido pseudopapilar del páncreas: Caso clínico. Rev Chil Cir. 2008;60:241-5.

15. Carreño L, Smok G, Villarroel M, Sanhueza V. Neoplasia sólida seudopapilar del páncreas: comunicación de 5 casos. Gastroenterol Hepatol. 2011;34:266-70. 
16. Venturelli FM, Carrasco CL, Del Pozo ML, Carcamo CI, Felmer OE, Ibaceta MC, et al. Solid-pseudopapillary tumor of the pancreas. Presentation of 3 clinical cases. Rev Gastroenterol Peru 2011;31:72-6.

17. Zúñiga J, Buchheister M, F. V. Doble tumor sólido pseudopapilar del páncreas. Rev Chil Cir 2011;63:13-4.

18. Min Kim S, Sun CD, Park KC, Kim HG, Lee WJ, Choi $\mathrm{SH}$. Accumulation of beta-catenin protein, mutations in exon-3 of the beta-catenin gene and a loss of heterozygosity of 5 q22 in solid pseudopapillary tumor of the pancreas. J Surg Oncol. 2006;94:418-25.

19. Kang CM, Kim HK, Kim H, Choi GH, Kim KS, Choi JS, et al. Expression of Wnt target genes in solid pseudopapillary tumor of the pancreas: a pilot study. Pancreas 2009;38:e53-9.

20. Tanaka Y, Kato K, Notohara K, Hojo H, Ijiri R, Miyake $\mathrm{T}$, et al. Frequent beta-catenin mutation and cytoplasmic/nuclear accumulation in pancreatic solid-pseudopapillary neoplasm. Cancer Res. 2001;61:8401-4.

21. Butte JM, Brennan MF, Gonen M, Tang LH, D’Angelica MI, Fong Y, et al. Solid pseudopapillary tumors of the pancreas. Clinical features, surgical outcomes, and longterm survival in 45 consecutive patients from a single center. J Gastrointest Surg. 2011;15:350-7.

22. Tognarini I, Tonelli F, Nesi G, Martineti V, Galli G, Gozzini A, et al. In vitro effects of oestrogens, antioestrogens and SERMs on pancreatic solid pseudopapillary neoplasm-derived primary cell culture. Cell Oncol. 2010;32:331-43.

23. Ozturk Y, Soylu OB, Gurcu B, Ortac R, Cakmakci H, Coker A. Solid pseudopapillary tumor of the pancreas as a cause of recurrent pancreatitis. Acta Gastroenterol Belg. 2008;71:390-2.

24. Belletrutti PJ, Allen PJ, Kurtz RC, DiMaio CJ. Education and imaging. Hepatobiliary and pancreatic: Recurrent pancreatitis caused by a solid pseudopapillary neoplasm of the pancreas. J Gastroenterol Hepatol. 2011;26:787.

25. Kim HH, Yun SK, Kim JC, Park EK, Seoung JS, Hur YH, et al. Clinical features and surgical outcome of solid pseudopapillary tumor of the pancreas: 30 consecutive clinical cases. Hepatogastroenterology 2011;58:1002-8.

26. Choi JY, Kim MJ, Kim JH, Kim SH, Lim JS, Oh YT, et al. Solid pseudopapillary tumor of the pancreas: typical and atypical manifestations. AJR Am J Roentgenol. 2006;187:W178-86.

27. Yu MH, Lee JY, Kim MA, Kim SH, Lee JM, Han JK, et al. MR imaging features of small solid pseudopapillary tumors: retrospective differentiation from other small solid pancreatic tumors. AJR Am J Roentgenol. 2010;195:1324-32.

28. Stoita A, Earls P, Williams D. Pancreatic solid pseudopapillary tumours - EUS FNA is the ideal tool for diagnosis. ANZ J Surg. 2010;80:615-8.

29. Kim CW, Han DJ, Kim J, Kim YH, Park JB, Kim SC. Solid pseudopapillary tumor of the pancreas: can malignancy be predicted? Surgery 2011;149:625-34.

30. Fais PO, Carricaburu E, Sarnacki S, Berrebi D, Orbach $\mathrm{D}$, Baudoin $\mathrm{V}$, et al. Is laparoscopic management suitable for solid pseudo-papillary tumors of the pancreas? Pediatr Surg Int. 2009;25:617-21.

31. Alvise C, Giovanni B, Despoina D, Roberto S, Gianluigi M, Micaela P, et al. Laparoscopic pancreatectomy for solid pseudo-papillary tumors of the pancreas is a suitable technique; our experience with long-term follow-up and review of the literature. Ann Surg Oncol. 2011;18:352-7.

32. Kooby DA, Gillespie T, Bentrem D, Nakeeb A, Schmidt MC, Merchant NB, et al. Left-sided pancreatectomy: a multicenter comparison of laparoscopic and open approaches. Ann Surg. 2008;248:438-46.

33. Fisher WE, Hodges SE, Silberfein EJ, Artinyan A, Ahern $\mathrm{CH}$, Jo E, et al. Pancreatic resection without routine intraperitoneal drainage. HPB (Oxford) 2011; 13:503-10.

34. Bassi C, Molinari E, Malleo G, Crippa S, Butturini G, Salvia R, et al. Early versus late drain removal after standard pancreatic resections: results of a prospective randomized trial. Ann Surg. 2010;252:207-14. 